\title{
XMM-Newton spectrum of the radio-loud quasar 3C 215: Slim accretion disk or SMBH binary
}

\author{
A. Mei ${ }^{1,2,3}$ and F. Tombesi ${ }^{2,4,5,6,7}$ \\ ${ }^{1}$ Gran Sasso Science Institute (GSSI), Viale Francesco Crispi 7, 67100 L'Aquila, AQ, Italy \\ e-mail: alessio.mei@gssi.it \\ 2 Department of Physics, University of Rome "Tor Vergata", Via della Ricerca Scientifica 1, 00133 Rome, Italy \\ 3 INFN-Laboratori Nazionali del Gran Sasso, 67100 L'Aquila, AQ, Italy \\ 4 Department of Astronomy, University of Maryland, College Park, MD 20742, USA \\ 5 NASA/Goddard Space Flight Center, Code 662, Greenbelt, MD 20771, USA \\ 6 INAF-Osservatorio Astronomico di Roma, Via Frascati 33, 00078 Monteporzio Catone, Italy \\ 7 INFN-Tor Vergata, Via della Ricerca Scientifica 1, 00133 Rome, Italy
}

Received 1 February 2021 / Accepted 28 July 2021

\begin{abstract}
Context. Radio-loud active galactic nuclei (RL AGN) exhibit very powerful jet emission in the radio band, while the radio-quiet (RQ) AGN do not. This RL-RQ dichotomy would imply a sharp difference existing among these two classes, however, modern theoretical models and observations suggest a common nuclear environment that is possibly characterized by different working regimes.

Aims. We explore the geometrical structure and mutual interactions of the innermost components of the broad line radio galaxy $3 \mathrm{C} 215$, with a particular focus on the accretion and ejection mechanisms involving the central supermassive black hole (SMBH). We compare these observational features with those of the RQ Seyfert 1 galaxies. Investigating their differences is aimed at improving our understanding of the jet launching mechanisms and devising an explanation for why this phenomenon is efficient only in a small fraction of all the AGNs.

Methods. Using high-quality data from a $\sim 60 \mathrm{ks}$ observation with XMM-Newton, we carried out a detailed X-ray spectral analysis of 3C 215 in the broad energy range of $0.5-10 \mathrm{keV}$. We modeled the spectrum with an absorbed double power-law model for the primary continuum, reprocessed by reflection from ionized and cold neutral material and modified by relativistic blurring. We also compared our results with those of earlier multi-wavelength observations.

Results. We obtained a primary continuum photon index from the corona, namely, $\Gamma_{1}=1.97 \pm 0.06$, along with evidence of a jet contribution, modeled as a power law with photon index of $\Gamma_{2} \simeq 1.29$. The reflector, which is possibly attributed to the accretion disk and portions of the broad-line region (BLR), is ionized $\left(\log \xi=2.31_{-0.27}^{+0.37} \mathrm{erg} \mathrm{s}^{-1} \mathrm{~cm}\right.$ ) and relatively distant from the SMBH $\left(R_{\mathrm{in}}>38 R_{\mathrm{g}}\right)$, where $R_{\mathrm{g}}=G M_{\mathrm{BH}} / c^{2}$ is the gravitational radius. The obscuring torus seems patchy, dust-poor, and inefficient, while the jet emission shows a twisted and knotted geometry. We propose three scenarios to describe the following characteristics: 1 . An ADAF state in the inner disk; 2. A slim accretion disk; and 3. A sub-pc SMBH binary system (SMBHB).

Conclusions. While the first scenario is not in agreement with the SMBH accretion regime, the slim disk scenario is consistent with the observational features of this radio galaxy, showing that $3 \mathrm{C} 215$ is similar to non-jetted AGNs, accreting at a high rate. Nonetheless, the first two scenarios are unable to account for the particular shape of 3C 215 jet emission. The SMBHB scenario seems to be in agreement with almost all 3C 215 observational features, but we are not able to unequivocally determine this source as a strong SMBHB candidate. A final determination will require further analysis.
\end{abstract}

Key words. galaxies: active - galaxies: nuclei - quasars: supermassive black holes - accretion, accretion disks

\section{Introduction}

It is now widely accepted that at the center of virtually all galaxies there resides a supermassive black hole (SMBH) with a mass in the range between $10^{6}$ and $10^{9} M_{\odot}$. Through the accretion of matter, it is able to efficiently convert gravitational energy, powering the plasma in its sphere of influence and, in relatively few cases $(\sim 1 \%)$, forming an active galactic nucleus (AGN). These sources are characterized by a high luminosity and broadband emission ranging from the radio band to the X-ray and $\gamma$-ray, as well as significant time variability. This places AGN among the brightest and most complex sources in the Universe (Padovani et al. 2017).

According to the unified model, the peculiar emission of $\mathrm{AGN}$ is produced as a result of the interaction between the cen- tral accreting SMBH and some fundamental components such as the accretion disk, hot corona, broad line region (BLR), and the obscuring torus and outflows, which are considered ubiquitous in AGN (Urry \& Padovani 1995). A small fraction of all the AGN $(\sim 10 \%)$ shows an intense emission in the radio band compared to the optical. Observations reveal that these radio-loud (RL) AGN host a powerful jet emission originating in the nuclear regions, while for the radio-quiet (RQ) AGN, this is several orders of magnitude weaker or absent (Padovani 2017).

In order to produce high energy photons and relativistic particles in jets, an interaction between the magnetic field and the accretion disk is thought to be taking place in close proximity to a quickly spinning SMBH (Blandford \& Znajek 1977; Ballantyne 2007); apparently, these conditions are only fulfilled in the RL sources. The exact jet-launching mechanisms are still 
unclear and continue to be a matter of debate, making RL AGN very interesting sources for observation and analysis. Nonetheless, this is a challenging task, given these sources intrinsically rare. Thus, for this purpose, the X-ray analysis of broad-line radio galaxies (BLRGs) constitute an important benchmark for the study of the emission and accretion mechanisms taking place in their innermost regions, near the SMBH. This class of RL AGN is relatively luminous in the X-rays and they are observed with an intermediate inclination angle that is large enough to avoid observing spectra that have been entirely dominated by the jet emission (as in the blazar class) and small enough to avoid obscuration from the torus.

By analyzing similarities and differences between BLRGs and their RQ counterparts, the Seyfert 1 galaxies, which show a very similar spectrum but an absence (or very faint) jet component, it is possible to explore what the jet production mechanisms might be and to test the so called RL-RQ dichotomy. RL AGN seem to have a smaller accretion rate (Panessa et al. 2007) and higher BH masses (Laor 2000; Chiaberge \& Marconi 2011) with respect to the RQ AGN. Moreover, RL AGNs are mostly hosted in elliptical or bulge-dominated galaxies, while RQ can be found both in spirals and elliptical galaxies (Floyd et al. 2004). This suggest a connection between RL AGNs and major merger events of the host galaxies during their evolution (Ravindranath et al. 2002; Chiaberge et al. 2015).

As pointed out by Eracleous et al. (2000), BLRGs show weaker X-ray reflection signatures with respect to Seyfert 1 galaxies, with narrow or non-detectable iron lines and weak Compton reflection hump, possibly due to a particular geometry of the corona-disk system. In fact, if there is absence of matter in the innermost region of the accretion disk (i.e., a truncated disk) or an advection dominated accretion flow, namely, an ADAF disk (Narayan \& Yi 1994, 1995; Narayan \& McClintock 2008; Abramowicz \& Fragile 2013), the solid angle over which the outer standard thin disk is illuminated is relatively small, leading to an overall weakening of the reflection features.

Ballantyne et al. (2002) state that also the reprocessing from a highly ionized accretion disk $\left(\xi \sim 4000 \mathrm{erg} \mathrm{cm} \mathrm{s}^{-1}\right)$ can suppress the reflected spectrum, going on to argue that this phenomenon is not strictly correlated to a particular geometry and that a standard thin accretion disk may also account for this.

There is also evidence that BLRGs have harder X-ray spectra with respect to their RQ counterparts (Grandi et al. 2006). Nevertheless Sambruna et al. (1999) showed only a weak indication of a flatter spectrum in a BLRG.

Two important parameters that can be of a pivotal importance in testing the radio-loud-radio-quiet dichotomy are the Eddington parameter $\lambda=L_{\text {bol }} / L_{\text {Edd }}$ (where $L_{\text {bol }}$ is the bolometric luminosity and $L_{\text {Edd }}$ is the Eddington luminosity) (Ballantyne 2007) and the dimensionless black hole spin parameter $a=$ $c J / G M^{2}$. Recent observations indicate a direct proportional relation between the jet power and the spin parameter, suggesting that rapidly spinning SMBHs are more likely to form powerful jets (Moderski et al. 1998).

Garofalo et al. (2010, 2014) propose a different theoretical framework, arguing that the jet formation mechanism is not triggered by the intensity of the SMBH rotation, but the mutual rotational directions between the black hole and the accretion flow, favoring the formation of jets in the case of retrograde rotation $(a<0)$. This may explain the observational evidence of a low fraction of RL AGN at low redshift. In addition, merger episodes, which cause an high accretion rate and retrograde flows, can explain how RL AGNs are formed and why they are commonly observed in elliptical galaxies that have a high proba- bility of having experienced at least one significant merger event in their past evolution.

The jet formation mechanism can be described by a disk + jet model linked to a jet duty-cycle. According to this model, the accretion disk, which is initially intact down to the innermost stable circular orbit (ISCO), is internally disrupted because of disk instabilities and ejects matter that constitutes the jet and outflows. After this, the accretion disk refills and the cycle starts again.

This model, proposed by Lohfink et al. (2013), is in agreement with their multi-epoch observations of a BLRG caught both in the ejection and refilling stages, making it possible to consider BLRGs observed in different stages of their jet cycle and exhibiting different observational features.

Recent models, supported by new X-ray observations, claim that the nuclear regions of BLRGs and Seyfert 1 are composed by very similar components that interact with the same physical phenomena but at different regimes (Ballantyne et al. 2014; Ursini et al. 2018a).

\section{2. $3 C 215$}

3C 215 is a BLRG with a cosmological redshift $z=0.412$, measured through optical emission lines. This source has been observed different times over the years, covering a large fraction of the electromagnetic spectrum, but with little information in the X-ray band. In the following, we describe the most important characteristics of 3C 215 obtained from the literature.

The 3C 215 host galaxy is elliptical (Lehnert et al. 1999) and it is probably interacting with a companion galaxy at $\sim 28 \mathrm{kpc}$, in a clustered environment of a radius $\sim 30$ arcsec containing at least 14 other galaxies (Márquez et al. 1999). Therefore, it is likely that during its lifetime, this galaxy experienced at least one major merger episode, which led the gas in the disk to be expelled or accreted (or both).

Tang et al. (2012) observed the optical/UV emission lines and measured their flux, equivalent width (EW), and full width at half maximum (FWHM) for broad and narrow emission lines in this wave-band, notably the $\mathrm{C}_{\mathrm{IV}}, \mathrm{Mg}_{\mathrm{II}}$, and $\mathrm{H} \beta$ emission lines. They computed three different estimates of the black hole mass $M_{\mathrm{BH}}$ through empirical mass scale relations (Vestergaard \& Peterson 2006; Vestergaard \& Osmer 2009), adopting the median value for the black hole mass $\log M_{\mathrm{BH}}=8.92 \pm 0.5$.

Labita et al. (2006) observed a sample of quasi-stellar objects (QSOs), including 3C 215, in the UV band with the Hubble Space Telescope (HST) measuring the $\mathrm{C}_{\mathrm{IV}}$ line width and the host galaxy luminosity. These authors computed the black hole mass using the $M_{\mathrm{BH}}-L_{\text {bulge }}$ relation (Bettoni et al. 2003) and by comparing this mass estimate with the virial theorem, they calculated the geometry factor of the BLR $f=1 / 3$, indicating a disk-like BLR geometry.

Decarli et al. (2008) further analyzed the previous sample, while additionally considering the $\mathrm{H} \beta$ line. Unfortunately, $3 \mathrm{C} 215$ was excluded from the sample because of its low signalto-noise ratio $(\mathrm{S} / \mathrm{N})$, but what they found for the sample is a stratified BLR, with an inflated disk-like region that produces the $\mathrm{C}_{\mathrm{IV}}$ emission line and an isotropic region that produces the $\mathrm{H} \beta$ emission line, similar to what was found by Labita et al. (2006) with the $\mathrm{C}_{\mathrm{IV}}$ line.

Decarli et al. (2010) analyzed the same sample with different observatories and obtained an independent black hole mass trough the virial theorem. They obtained the same $M_{\mathrm{BH}}$ estimate 


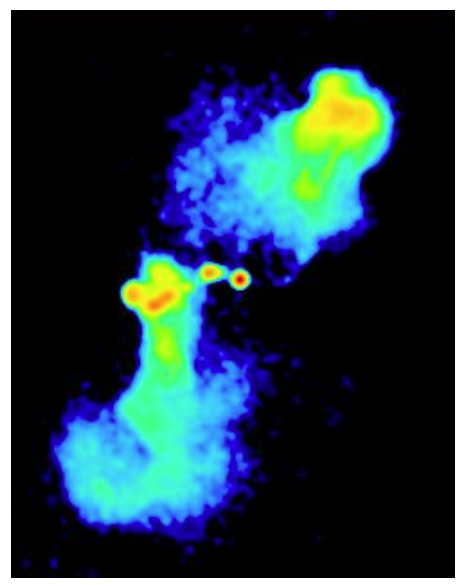

Fig. 1. VLA A+B image of 3C 215 at $4860 \mathrm{MHz}$ (Bridle et al. 1994).

of Tang et al. (2012), so this is the value that we will consider in this work.

Runnoe et al. (2013) carried out a SED analysis from the IR to the X-ray band, obtaining important parameter values related to this source, such as the bolometric luminosity, $\log L_{\mathrm{bol}} \simeq$ $45.77 \mathrm{erg} \mathrm{s}^{-1}$, the spectral index between the optical and the X-ray band, $\alpha_{\mathrm{ox}} \simeq-1.04$, the observable ratio between mid-IR and bolometric luminosity, $\log R \simeq-1.521$, and the obscuring torus covering fraction, $c \simeq 64 \%$, which is estimated using the IR flux.

Reeves \& Turner (2000, R\&T00) and Hardcastle et al. (2006, H06) both carried out with different telescopes an X-ray analysis of 3C 215 in the $2-10 \mathrm{keV}$ band, but with the low $\mathrm{S} / \mathrm{N}$ data available to them, they could only measure the luminosity in the X-ray band, $L_{\mathrm{X}}$, and the photon index, $\Gamma$. Their work is discussed in more detail in Sect. 5.3.

The radio band observations of $3 \mathrm{C} 215$ are ambiguous (Bridle et al. 1994; Gilbert et al. 2004; Fernini 2007). This source lies in a highly dense clustered environment and shows a knotty and twisted jet, tilted by almost $90^{\circ}$ with respect to the lobes (see Figs. 1 and 2).

The plume structure in the southern lobe is more similar to a Fanaroff-Riley (FR) I source, while the northern lobe resembles FR II features. The particular environment of 3C 215 is probably one of the reasons behind this particular behavior and it can interfere with the jet at scales of $\sim 10 \mathrm{kpc}$. However, to account for this complex structure, there have to be several additional phenomena taking place in the inner region of the AGN where the jet is formed, such as a warped accretion disk or a secondary $\mathrm{SMBH}$.

\section{Data reduction}

3C 215 was observed with XMM-Newton in April 17-18, 2012. In this work, in order to acquire a better $\mathrm{S} / \mathrm{N}$, we used data from the EPIC-pn, EPIC-MOS1 and EPIC-MOS2 detectors with total exposures of $59.6 \mathrm{ks}$ and $61.2 \mathrm{ks}$ for the pn and MOS cameras, respectively. The data reduction and analysis were carried out using the SAS standard procedure. We checked for the presence of soft proton flaring events in the dataset, with energies between $E=10-12 \mathrm{keV}$. We produced a light curve in the same energy range, excluding all the time intervals with a count rate that exceeds the values 0.7, 0.3 and 0.25 for the EPIC-pn, MOS1, and MOS2 datasets, respectively.

Afterwards, we defined a circular region that encircles the source, with radii of $40^{\prime \prime}$ and $44^{\prime \prime}$ in the EPIC-pn and MOS

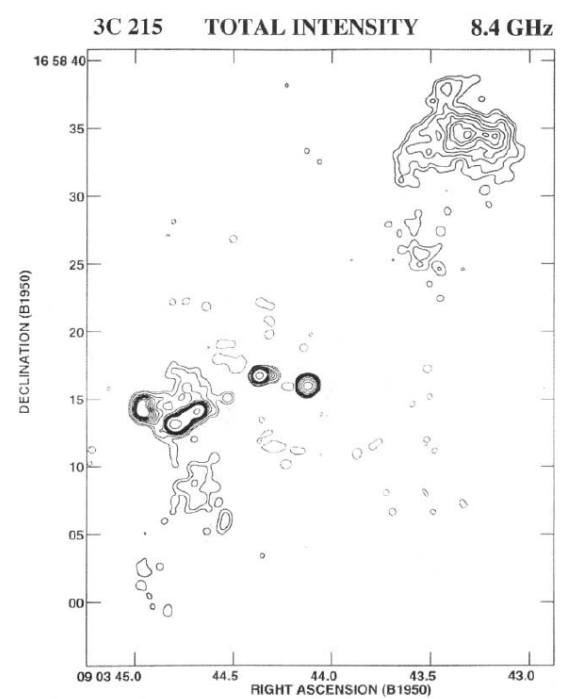

Fig. 2. VLA B contour map of 3C 215 at $8.4 \mathrm{GHz}$ (Fernini 2007).

CCDs, respectively. We repeated the same procedure with the background region, considering different portions of the same CCDs with a uniformly distributed number of photons for each pixel. In addition, we tested the presence of pile-up effects, excluding data contamination.

Using the SAS tools we produced redistribution matrix files (RMFs), ancillary response files (ARFs), and cleaned X-ray spectra in the energy range of $E=0.5-10 \mathrm{keV}$, grouped with a minimal threshold of 25 photons per bin.

\section{Spectral analysis}

To analyze the spectrum obtained through the data reduction, we used the package XSPEC (v. 12.10.1) from the software Heasarc (v. 6.26.1), which allowed us to analyze the spectrum using a standard spectral fitting method with $\chi^{2}$ statistics. The errors reported in this analysis are computed with a $1 \sigma$ confidence level, while the upper and lower limits are reported at a $90 \%$ confidence level.

Since we are using a combined spectrum produced from data from three different cameras, we took into account the crosscalibration differences between the EPIC-pn and EPIC-MOS devices through a cross-calibration parameter, $k$. Fixing $k=1$ for the EPIC-pn spectrum, we found from the EPIC-MOS spectral fit, $k_{\mathrm{mos}}=1.011$, which is henceforth considered fixed. This indicates a difference of only $\sim 1 \%$ in the absolute flux between the EPIC-pn and MOS cameras, and this is consistent with the latest ESA calibration.

\subsection{Absorbed power-law}

Because of the Compton scattering interaction between the accretion disk photons and the hot corona, the X-ray spectrum of this source can be modeled as a power-law with slope given by the photon index $\Gamma$, which is absorbed by the interstellar medium (ISM) in the Milky Way with a cross-section given by the Tuebingen-Boulder model (Wilms et al. 2000), called TBabs in XSPEC. To compute the ISM column density, we used the $\mathrm{nH}$ tool provided by Heasarc, which estimates $N_{\mathrm{H}}=3.5 \times 10^{20} \mathrm{~cm}^{-2}$ for 3C 215. The overall model fit, which in XSPEC notation is TBabs*zpowerlw, has an $\chi^{2} /$ d.o.f. $=742.83 / 711$, providing a good starting point for the spectral analysis. 


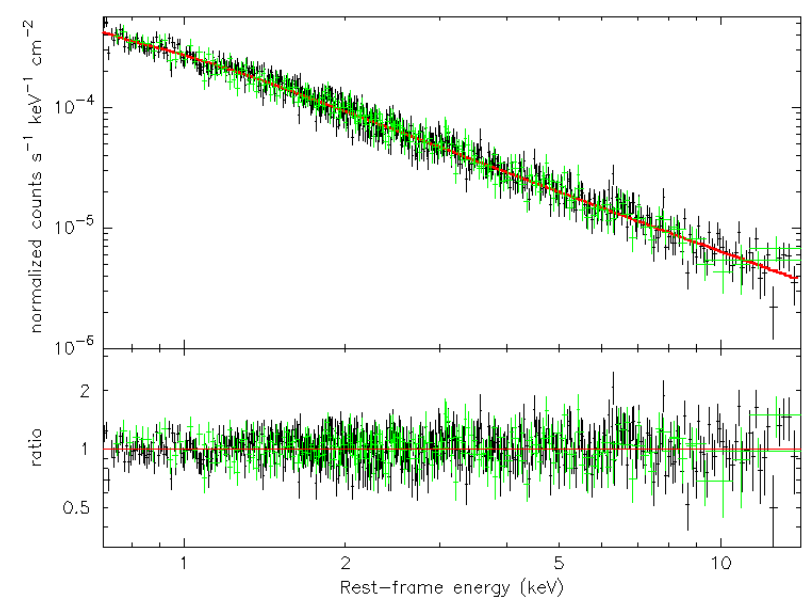

Fig. 3. X-ray spectrum (upper panel) and data to model ratio (lower panel), using the XSPEC notation, TBabs* (zpowerlw+zpowerlw) for pn and MOS data (in black and green, respectively). Model is shown with the red line.

\subsection{Double absorbed power law}

As discussed in Sect. 2, 3C 215 shows a strong jet emission. In order to explore the possible contribution of the jet in the X-ray spectrum, we added another power-law in the overall model, TBabs* (zpowerlw+zpowerlw) in the XSPEC notation. The fit provides two different values for the two photon indices, one soft $\left(\Gamma_{1}=1.97 \pm 0.06\right)$ and one hard $\left(\Gamma_{2}=1.29 \pm 0.15\right)$, with $\chi^{2} /$ d.o.f. $=729.36 / 710$. Thus, we fixed the jet power-law component to its best-fit value because having two free powerlaw slopes was causing problems when performing spectral fitting, as the fit routine was often switching between the two slopes. While the first power-law is consistent with the one expected from inverse Compton scattering in the disk-corona system, the second one is similar to the one observable in flat spectrum radio quasars (FSRQs) that, conversely, are dominated by the jet. The presence of both coronal and jet emission can be expected in the class of broad-line radio galaxies, given their intermediate inclination (Grandi et al. 2006; Kataoka et al. 2011; Tazaki et al. 2013; Bostrom et al. 2014). Nonetheless, the jet component is subdominant, with a luminosity of $\lesssim 25 \%$ for the corona in the $0.5-10 \mathrm{keV}$ spectrum, with luminosities (in cgs units) of $\log L_{\text {jet }}^{0.5-2 \mathrm{keV}}=43.81_{-0.10}^{+0.07}$ and $\log L_{\text {jet }}^{2-10 \mathrm{keV}}=44.35_{-0.10}^{+0.07}$ smaller than the one related to the corona emission, namely, $\log L_{\text {corona }}^{0.5-2 \mathrm{keV}}=44.44 \pm 0.02$ and $\log L_{\text {corona }}^{2-10 \mathrm{keV}}=44.53 \pm 0.05$. The significant improvement of the $\chi^{2}$ with respect to the single power-law model $\left(\Delta \chi^{2}=13.47\right.$ with one d.o.f. less, corresponding to $\left.P_{\text {jet }}=99.97 \%\right)$, leads us to include the presence of the jet emission in the X-ray spectrum of 3C 215.

\subsection{Modeling the emission and absorption features}

In the ratio plot in Fig. 3, there are hints of an emission line at $\sim 6.5 \mathrm{keV}$, likely associated with Fe $\mathrm{K}$ emission line and a possible absorption feature at $\sim 9 \mathrm{keV}$. We modeled these emission and absorption features using Gaussian profiles in the source rest frame, in XSPEC zgauss, with width $\sigma_{\mathrm{g}}$ and peak energy $E_{\mathrm{g}}$. For the emission line, the fit of the overall model, that in XSPEC notation is TBabs*(zgauss+zpowerlw+zpowerlw), provided the parameter values $E_{\mathrm{g}}=6.53_{-0.08}^{+0.11} \mathrm{keV}, \sigma_{\mathrm{g}}<0.41 \mathrm{keV}$, photon indices, $\Gamma_{1}=1.95 \pm 0.06$ and $\Gamma_{2}=1.29$, and equivalent width, $\mathrm{EW}_{\mathrm{g}}=74_{-30}^{+28} \mathrm{eV}$, the latter obtained with the XSPEC command eqwidth (see Fig. 4). Using the Fisher test we derive
Table 1. Best fit-value of the parameters for the model TBabs* (zgauss+zpowerlw+zpowerlw).

\begin{tabular}{|c|c|c|}
\hline Parameter & Value & Units \\
\hline \multicolumn{3}{|c|}{ Galactic absorption } \\
\hline$N_{\mathrm{H}^{*}}$ & 3.50 & $\times 10^{20} \mathrm{~cm}^{-2}$ \\
\hline \multicolumn{3}{|c|}{ Power-law 1 (corona) } \\
\hline$\Gamma$ & $1.95 \pm 0.06$ & - \\
\hline$N_{\mathrm{pl}}$ & $4.11_{-0.17}^{+0.19}$ & $\times 10^{-4} \frac{\text { counts }}{\mathrm{cm}^{2} \mathrm{~s} \mathrm{keV}}$ \\
\hline$z *$ & 0.412 & - \\
\hline \multicolumn{3}{|c|}{ Power-law 2 (jet) } \\
\hline$\Gamma *$ & 1.29 & - \\
\hline$N_{\mathrm{pl}}$ & $8.15_{-2.06}^{+1.77}$ & $\times 10^{-5} \frac{\text { counts }}{\mathrm{cm}^{2} \mathrm{~s} \mathrm{keV}}$ \\
\hline$z *$ & 0.412 & - \\
\hline \multicolumn{3}{|c|}{ Gaussian emission line } \\
\hline$E_{\mathrm{g}}$ & $6.53_{-0.08}^{+0.11}$ & $\mathrm{keV}$ \\
\hline$\sigma_{\mathrm{g}}$ & $<0.41$ & $\mathrm{keV}$ \\
\hline $\mathrm{EW}_{\mathrm{g}}$ & $74_{-30}^{+28}$ & $\mathrm{eV}$ \\
\hline$N_{\mathrm{g}}$ & $1.87_{-0.73}^{+0.99}$ & $\times 10^{-6} \frac{\text { counts }}{\mathrm{cm}^{2} \mathrm{~s} \mathrm{keV}}$ \\
\hline
\end{tabular}

Notes. $N_{\mathrm{pl}}$ is the normalization of the power-law, i.e. the flux at $1 \mathrm{keV}$. $N_{\mathrm{g}}$ is the normalization of the Gaussian profile, i.e. the total flux of the line. The starred parameters are the ones kept frozen during the fit. Upper and lower limits are calculated at a $90 \%$ confidence level.

a confidence level of $P_{\mathrm{em}}=98.7 \%$ for the detection of the emission line. This model provides $\chi^{2} /$ d.o.f. $=720.52 / 707$. We carried out the same analysis also for the possible absorption line with fixed width, showing $\chi^{2} /$ d.o.f. $=716.19 / 705$, while the relative Fisher test provided a probability of $P_{\mathrm{abs}}=89 \%$, which led us to discard the presence of this absorption feature on statistical grounds. The best-fit values of the parameters are shown in Table 1.

\subsection{Reflection from ionized material}

The peak energy, $E>6.4 \mathrm{keV}$, of the iron emission line suggests that the reflector is made by different ionized iron species, and this phenomenon can be interpreted as a reflection from ionized gas in the accretion disk. To account for this process, we used the additive model xillver (García et al. 2013), which can describe the reflected spectrum of the radiation that is reprocessed in an ionized accretion disk, requiring that the incident flux is generated by a power-law spectrum, as in the case of the Comptonized emission from an hot corona in the AGN environment.

In this model, the ionization parameter, $\xi=L_{\text {ion }} / n r^{2}$, is introduced, where $L_{\text {ion }}$ is the luminosity of the incident ionizing radiation in the energy range of $E=13.6 \mathrm{eV}-13.6 \mathrm{keV}$, while $n$ and $r$ are the density of the reflector and its distance from the ionizing source, respectively. This model is also dependent on the inclination angle of the line of sight, $i$. We kept this value fixed at $i=30^{\circ}$, which is a typical value for a BLRG, since the fit is unable to be constrained it if it is set free to vary.

In addition, we used a Gaussian smoothing profile with an energy-independent width, in XSPEC gsmooth, in order to consider the possibility of velocity broadening of the line in addition to the observed broadening due to the presence of emission from a range of iron ions. The overall model fit, 


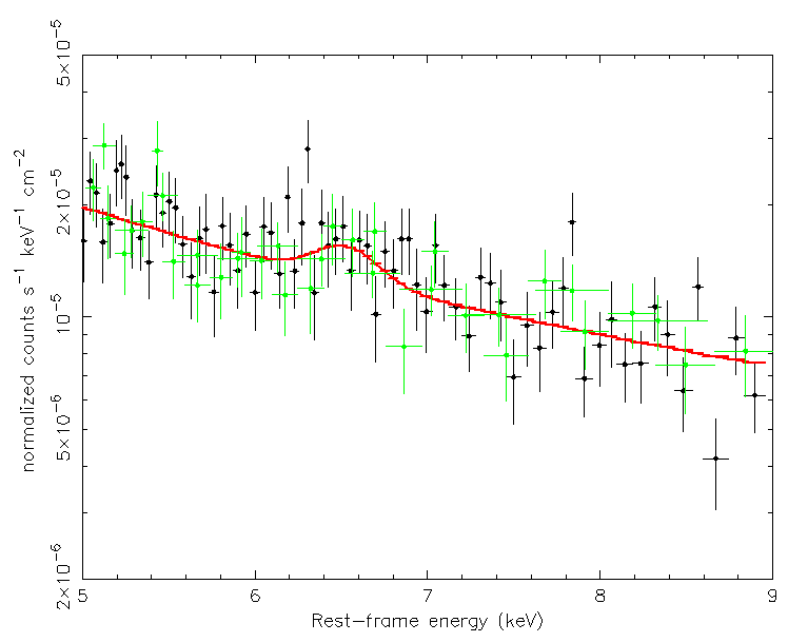

Fig. 4. Zoomed pn and MOS data (in black and green, respectively) and model (in red) in the $5-9 \mathrm{keV}$ band including a Gaussian emission line at $E \simeq 6.53 \mathrm{keV}$ in the model TBabs*(zgauss+ zpowerlw+zpowerlw), using XSPEC notation.

in XSPEC TBabs*(zpowerlw+gsmooth*xillver), provides a width upper limit of $\sigma_{\text {ion }}<82 \mathrm{eV}$, ionization of $\log \xi=$ $3.92_{-0.15}^{+0.13}$, and corona photon index of $\Gamma=1.82_{-0.03}^{+0.02}$. The value $\chi^{2} /$ d.o.f. $=728.75 / 709$ is comparable with that of the double power law model.

\subsection{The relxill model}

We find an excess of width of the emission line when considering both phenomenological and xillver models, with a relatively large FWHM of $\sim 10000 \mathrm{~km} \mathrm{~s}^{-1}$, which would suggest a location relatively close to the SMBH. Here, we test the hypothesis that the width of the line is due to relativistic effects from reflection onto accretion disk using the model relxill (García et al. 2014; Dauser et al. 2014), which takes into account the special and general relativity effects shaping the reflected emission line profile. This model introduces new parameters, namely, the dimensionless spin parameter, $a=J c / G M^{2}$ (kept fixed at the maximum value $a=0.998$ ), the distance between the inner radius of the reflector and the $\mathrm{SMBH}, R_{\mathrm{in}}$, the distance of the outer radius, $R_{\text {out }}$ (kept fixed at the standard value $R_{\text {out }}=10^{3} R_{\mathrm{g}}$ ), and the reflection fraction, $R_{\mathrm{f}}$, namely, the ratio between the reflected and direct emission. The best-fit values relative to this model, in XSPEC nomenclature TBabs* (zpowerlw + relxill), are $\Gamma_{1}=$ $1.93 \pm 0.02, \log \xi=2.31_{-0.27}^{+0.37}, R_{\mathrm{in}}>38 R_{\mathrm{g}}, R_{\mathrm{f}}=0.09 \pm$ 0.04 with $\chi^{2} /$ d.o.f. $=722.47 / 708$, showing an improvement of $\Delta \chi^{2}=6.28$ with 1 d.o.f. less and confidence level $P_{\text {relxill }}=$ $98 \%$ with respect to the xillver model. The best-fit value of the parameters are shown in Table 2. We decided to choose TBabs* (zpowerlw+relxill) as our best-fit model, as it performs well in characterizing the X-ray spectrum of 3C 215.

\subsection{Neutral reflection from cold material}

Despite establishing the best-fit model for the spectrum of 3C 215 in Sect. 4.5, we tested an additional model component in order to verify the presence or absence of neutral Compton reflection from a distant, cold, and optically thick material (such as the putative AGN torus). We used the model pexmon (Nandra et al. 2007). We added this model as a new component
Table 2. Best fit-value of the parameters for the model TBabs*(zpowerlw+relxill).

\begin{tabular}{lcc}
\hline \hline Parameter & Value & Units \\
\hline Galactic absorption & & \\
\hline$N_{\mathrm{H}}$ & 3.50 & $\times 10^{20} \mathrm{~cm}^{-2}$ \\
\hline Relativistic effects & & \\
\hline$\Gamma$ & $1.91 \pm 0.02$ & - \\
$z^{*}$ & 0.412 & - \\
$N_{\mathrm{rel}}$ & $4.77 \pm 0.11$ & $\times 10^{-6} \frac{\mathrm{counts}}{\mathrm{cm}^{2} \mathrm{~s} \mathrm{keV}}$ \\
$\log \xi$ & $2.31_{-0.27}^{+0.37}$ & $\mathrm{erg} \mathrm{s}^{-1} \mathrm{~cm}$ \\
$a *$ & 0.998 & - \\
$R_{\text {in }}$ & $>38$ & $R_{\mathrm{g}}$ \\
$R_{\mathrm{out}} *$ & $10^{3}$ & $R_{\mathrm{g}}$ \\
$R_{\mathrm{f}}$ & $0.09 \pm 0.04$ & - \\
$i *$ & 30 & $\circ$ \\
$\beta *$ & 3 & - \\
$E_{\mathrm{cut}} *$ & 300 & $\mathrm{keV}$ \\
$A_{\mathrm{Fe}} *$ & 1 & $A_{\odot}$ \\
\hline Power-law 2(jet) & & - \\
\hline$\Gamma *$ & 1.29 & - \\
$N_{\mathrm{pl}} *$ & 8.15 & $\times 10^{-5} \frac{\mathrm{counts}}{\mathrm{cm}^{2} \mathrm{~s} \mathrm{keV}}$ \\
$z *$ & 0.412 & - \\
\hline
\end{tabular}

Notes. $N_{\text {rel }}$ is the normalization in relxill (Dauser et al. 2016). $E_{\text {cut }}$ is the energy of the observed cut-off; $A_{\mathrm{Fe}}$ is the reflector iron abundance in solar units; $\beta$ is the coronal emissivity slope, which is parameterized as a single power law. The starred parameters are the ones kept frozen during the fit. Upper and lower limits are calculated at a 90\% confidence level.

to the best-fit model shown in Table 2. Since we are interested in the neutral $\mathrm{Fe} \mathrm{K} \alpha(E=6.4 \mathrm{keV})$ equivalent width, we freeze all the parameters, except for $R_{\mathrm{pex}}$, in order to derive limits on the presence of neutral reflection. We obtained a value $R_{\text {pex }} \simeq 0$ for the neutral reflection fraction. We also computed the $\mathrm{Fe} \mathrm{K} \alpha$ EW modeling the line with a Gaussian profile, fixing the energy peak $E=6.4 \mathrm{keV}$ and line width $\sigma=0$ and adding this new component to the ionized iron emission line model. We obtained an upper limit $\mathrm{EW}_{\mathrm{K} \alpha}<26 \mathrm{eV}$. In order to explore the jet effect on this estimate, we performed the same calculation eliminating data and the second power-law in the model (the one describing the jet emission) and we found that $\mathrm{EW}_{\mathrm{K} \alpha}<41 \mathrm{eV}$. The upper limit is computed by fixing the line intensity with its bestfit value plus the associated intensity error. This is an indication of an absent or weak neutral reflection features, whose implications for the putative AGN torus and SMBH feeding is discussed in Sect. 5.

\section{Results and discussion}

The best-fit model we found in the spectral analysis in Sect. 4 describes the X-ray spectrum of 3C 215 as an absorbed double power-law with a tentative detection of an iron emission line peaking at $E \sim 6.53 \mathrm{keV}$. The shape and ionization of the emission line is affected by the interaction with an ionized reflector and relativistic blurring, and both are taken into account in the model. 
We computed the ionizing continuum luminosity, $L_{\text {ion }} \simeq$ $1.38 \times 10^{45} \mathrm{erg} \mathrm{s}^{-1}$, modeling the ionizing spectrum as a single power-law between $E=13.6 \mathrm{eV}-13.6 \mathrm{keV}$, without the contribution of the jet and using the XSPEC command clumin. We also calculated the X-ray luminosity, $L_{X}=(5.49 \pm 0.13) \times$ $10^{44} \mathrm{erg} \mathrm{s}^{-1}$, defined as the spectrum luminosity in an energy range between $E=2-10 \mathrm{keV}$ and considering both the contributions of the corona and the jet.

Previous observation of this source in the optical/UV band provided an estimate of the central SMBH mass $\log \left(M_{\mathrm{BH}} / M_{\odot}\right)=8.92 \pm 0.5$ (Tang et al. 2012). From this value, we are able to estimate the Eddington luminosity $L_{\text {Edd }}=$ $1.3 \times 10^{38} M_{\mathrm{BH}} / M_{\odot} \mathrm{erg} \mathrm{s}^{-1}$ and the gravitational radius $R_{\mathrm{g}}=$ $G M_{\mathrm{BH}} / c^{2}$, which will be used as an unit of measure for distances in the nuclear region on this AGN. Their values in log scale and cgs units are $\log L_{\mathrm{Edd}}=47.03 \pm 0.5$ and $\log R_{\mathrm{g}}=14.9 \pm 0.5$, respectively. By merging the information obtained from our analysis with the data presented in the literature, it is possible to derive a more detailed description of this AGN and of its nuclear environment.

\subsection{Jet contribution in the $X$-ray spectrum}

The $0.5-10 \mathrm{keV}$ spectrum of $3 \mathrm{C} 215$ shows a jet contribution in the form of a flat power law $(\Gamma \sim 1.29)$ together with a second steep power law $(\Gamma \sim 1.95)$ likely associated with the Inverse Compton emission originated in the corona. As discussed in the previous section, the contribution of the jet power law to the overall X-ray spectrum is subdominant with respect to the corona emission $\left(L_{\text {jet }} \lesssim 25 \%\right.$ ), in accordance with other BLRG observations (Grandi \& Palumbo 2007). This result is also in agreement with 3 C 215 mixing parameter estimate $\eta \simeq 0.034$ (Kataoka et al. 2011), computed through SED fitting and defined as the ratio between non-thermal and thermal emission.

The jet component seems to connect this source to blazars, in particular to FSRQs, which have harder photon indices in the $\mathrm{X}$-ray band on average, as compared to the blazar class overall (Sambruna et al. 1994; Comastri et al. 1997; Sambruna 1997; Fan et al. 2012). Moreover, the observed optical broad emission lines (Tang et al. 2012), the high luminosity and flat radio spectrum (shown in 3C 215 SED reported in the NASA/IPAC Extragalactic Database) further corroborates this hypothesis.

The joint observation of a steep Seyfert-like spectrum and a FSRQ spectrum in the X-ray band leads us to assume an intermediate inclination angle for this source, as expected for BLRGs. Nonetheless, these particular sources strongly challenge the bounds of the unified model, since there are observations of BLRGs with a visible jet contribution (e.g. Bostrom et al. 2014), while other BLRGs do not exhibit the jet component in the X-ray band (e.g. Ronchini et al. 2019), leading to assume that also in the BLRG class there exist other sub-classes with different observative features that may not be explained only by different observational angles, since it is still unclear at which inclination angle a BLRG stops being "blazar-like" and starts to be more "Seyfert-like".

\subsection{Ionized reflector}

Our data analysis of 3C 215 shows that the reflector, which interacts with the ionizing primary radiation from the hot corona, is placed at distance $R_{\text {in }} \gtrsim 38 R_{\mathrm{g}}$ from the $\mathrm{SMBH}$, which is relatively far compared to the ISCO value of $R_{\text {in }} \sim 1 R_{\mathrm{g}}$ expected for an AGN with a SMBH spin of $a \sim 1$. It is also ionized, with an ionization parameter $\xi \simeq L_{\text {ion }} / n R_{\text {in }}^{2} \simeq 204 \mathrm{erg} \mathrm{s}^{-1} \mathrm{~cm}$. Inverting the relation, we can obtain an upper limit for the reflector particle density $n \lesssim 10^{10} \mathrm{~cm}^{-3}$.

The density estimated in this calculation is low for an internal region of the accretion disk, whose density is expected to be of the order of $\sim 10^{15} \mathrm{~cm}^{-3}$. The density of the reflector seems to be more in agreement with the BLR, with a typical density of $\sim 10^{10} \mathrm{~cm}^{-3}$, or with an outer ionized layer of the accretion disk.

In previous observations in the optical/UV band of $3 \mathrm{C} 215$, there were hints of a stratified BLR with a disk-like geometry for the region producing the $\mathrm{C}_{\mathrm{IV}}$ emission line (Labita et al. 2006). Tang et al. (2012) provided the FWHM of some broad optical emission lines of this source, such as $\mathrm{C}_{\mathrm{IV}}$ and $\mathrm{H} \beta$, with values $F W H M\left(C_{\mathrm{IV}}\right)=5605_{-85}^{+90} \mathrm{~km} \mathrm{~s}^{-1}$ and $F W H M(\mathrm{H} \beta)=$ $6760_{-505}^{+500} \mathrm{~km} \mathrm{~s}^{-1}$, respectively.

We observe that the $\operatorname{FWHM}(\mathrm{H} \beta)$ value is slightly larger than the $\mathrm{C}_{\mathrm{IV}}$ one. From standard BLR stratification models, we would expect the opposite, with the more ionized line produced more internally, hence, with an higher FWHM, related to the velocity dispersion. This evidence can be explained considering that, since the line of sight is tilted with respect to the equatorial plane, we probably observe an $\mathrm{H} \beta$ outflowing source, which has a higher velocity dispersion along the line of sight, hence, a higher FWHM, even if the source projected on the equatorial plane is more external than the $\mathrm{C}_{\mathrm{IV}}$ one, which, in contrast, has a disk-like geometry.

Considering the reverberation mapping relation $R_{\mathrm{BLR}}=$ $G M_{\mathrm{BH}} / F W H M^{2}$, the disk-like portion of the BLR can cover the accretion disk surface at distances of $\gtrsim 10^{2} R_{\mathrm{g}}$ thus contributing to the reflection phenomenon that generates the reflected spectrum of $3 \mathrm{C} 215$ in the X-ray band. For distances of $R \lesssim 38 R_{\mathrm{g}}$ the ionizing flux from the hot corona can fully ionize elements lighter than iron, thereby excluding emission lines in the opti$\mathrm{cal} / \mathrm{UV}$ band. This may, at least partially, explain why there is no presence of observable reflection features in the SMBH neighborhood. At the distance of $R_{\mathrm{BLR}}$, where the bulk reflection from the BLR is taking place, we expect a reflector less ionized with respect to the reflection in the inner portion of the disk, where $\log \xi=2.31_{-0.27}^{+0.37} \mathrm{erg} \mathrm{s}^{-1} \mathrm{~cm}$. From the calculations, considering $n \sim 10^{10} \mathrm{~cm}^{-3}$ and $R_{\mathrm{BLR}} \sim 4 \times 10^{2} R_{\mathrm{g}}$, calculated as the mean value of the two emission lines, we obtain:

$\xi_{\mathrm{BLR}}=\frac{L_{\mathrm{ion}}}{n R_{\mathrm{BLR}}^{2}} \sim 1.6 \mathrm{erg} \mathrm{s}^{-1} \mathrm{~cm} \ll 204 \mathrm{erg} \mathrm{s}^{-1} \mathrm{~cm}$.

This evidence suggests a standard optically thick and geometrically thin structure in the outer part of the accretion disk of 3C 215, while the inner part could be a truncated disk or a composite disk, whose inner part is, in turn, radiatively inefficient; namely, it is in an ADAF-like state or, alternatively, a puffed-up slim disk.

\subsection{Long-term time variability of the $X$-ray spectrum}

In order to better describe this AGN, it is important to compute the Eddington parameter, $\lambda=L_{\mathrm{bol}} / L_{\mathrm{Edd}}$, where $L_{\mathrm{bol}}$ is the bolometric luminosity. We were able to estimate $L_{\text {bol }}$ starting from $L_{\mathrm{X}}$ through the bolometric correction $K_{\mathrm{X}}=L_{\mathrm{bol}} / L_{\mathrm{X}}$ parametric equation derived by Duras et al. (2020). Using this procedure we computed the Eddington parameter $\lambda$ for our observation and for the previous X-ray observations of 3C 215 in the energy band of $E=0.5-10 \mathrm{keV}$, carried out by R\&T00 and H06. Using observations of different satellites, these authors managed to estimate the X-ray luminosity $L_{\mathrm{X}}$. The results are summarized in Table 3. 
Table 3. Information and parameter values concerning the observations of 3C 215 in Reeves \& Turner (2000, R\&T00), in Hardcastle et al. (2006, H06) and in our spectral analysis.

\begin{tabular}{lccc}
\hline \hline & R\&T00 & H06 & This work \\
\hline Telescope & ASCA & Chandra & XMM \\
Obs. year & 1995 & 2004 & 2012 \\
$L_{\mathrm{X}}\left[10^{44} \frac{\mathrm{erg}}{\mathrm{s}}\right]$ & 17.20 & 6.92 & $5.49 \pm 0.13$ \\
$K_{\mathrm{X}}$ & 34.63 & 26.36 & $24.97 \pm 0.22$ \\
$L_{\text {bol }}\left[10^{46} \frac{\mathrm{erg}}{\mathrm{s}}\right]$ & 5.96 & 1.82 & $1.37 \pm 0.04$ \\
$\lambda$ & 0.54 & 0.17 & $0.13_{-0.09}^{+0.28}$ \\
\hline
\end{tabular}

Comparing these multi-epoch estimates, we observed variability of the X-ray spectrum on time-scales of around eight years. Namely, $L_{X}$ decreases, producing a recent spectrum dimmer with respect to the one observed $\sim 17$ years before our observation. Evidences of this kind of variability in the X-ray spectrum of AGNs were already found in Seyfert 1 galaxies (Noda et al. 2014, 2016), with timescales from weeks to years. There is no evidence of a jet contribution in the long-term variability of this source, both because it is not dominant in the X-ray band and because past observations were not precise enough to evidence this feature.

This phenomenon can be attributed to a change of the accretion rate (Noda et al. 2014). An efficiently accreting SMBH is very luminous and forms an optically thick and geometrically thin disk up to the ISCO and a subdominant, if present, internal ADAF state. If the accretion rate and, consequently, the luminosity decrease, as in this case, the ADAF state is no more subdominant in the internal region, the disk emissivity drops and the dominance of the hot corona in the overall X-ray emission produces an harder spectrum. Based on the same principle, Noda \& Done (2018) explain the changing-look phenomenon of the source Mrk 1018, which, across a time span of $\sim 8 \mathrm{yr}$ switches from a Seyfert 1 galaxy to a Seyfert 2. They state that the variation in the accretion rate is driven mainly by two combined factors, previously observed in BH binary systems: 1 . The evaporation or condensation of the inner disk in an advectiondominated hot flow causing a respective decrease or increase in the luminosity by a factor of $\sim 2-4$; and 2 . Thermal front propagation due to the $\mathrm{H}$-ionization instability in the disk.

In the case of $3 \mathrm{C} 215$, we observe a variability of almost a factor of $\sim 3$ from 1995 to 2012, which is more comparable to the classic X-ray variability of AGNs rather than a changing-look phenomenon, where luminosity drops by at least an order of magnitude. Therefore, the observational evidences of 3C 215 suggest an evolution of the inner structure of the AGN, consistent with the luminosity drop caused by the evaporation of the inner thin disk, as proposed by Noda \& Done (2018). However, we must note that the Eddington parameter estimated through our analysis is not completely in agreement with the values expected by this scenario, which is expected to be $\lambda \sim 1 \%$. This leads us to believe that, with respect to the other sources, the contribution of the standard outer disk in $3 \mathrm{C} 215$ is more relevant, questioning the presence and the importance of the ADAF in the inner disk.

\subsection{Lack of neutral absorption and reflection from the torus}

We want to underline the role of the putative obscuring torus that surrounds the nuclear regions of $3 \mathrm{C} 215$. It is typically made by optically thick material that interacts with the X-ray emission from the nuclear region through absorption and Compton scattering, giving important information about its geometry. Because of its distance from the SMBH, typically of $\sim$ pc scale, it is too cold to be ionized, hence we test its presence analyzing the neutral reflection component of the spectrum. From the data analysis, we find an upper limit for the equivalent width of the neutral $\mathrm{Fe}$ $\mathrm{K} \alpha$ line without jet contribution, namely, $\mathrm{EW}_{\mathrm{K} \alpha} \lesssim 41 \mathrm{eV}$, indicating a weak interaction between the primary $\mathrm{X}$-ray radiation and the torus, which gives a minimal contribution to the overall $\mathrm{X}$-ray spectrum. Theoretical models associated to such a weak or absent iron line feature a disrupted and inefficient obscurer with a patchy geometry, low column density $N_{\mathrm{H}}$ (with an upper limit $N_{\mathrm{H}}<5 \times 10^{22} \mathrm{~cm}^{-2}$ ) (Ikeda et al. 2009; Murphy \& Yaqoob 2009), and poor presence of dust (Gohil \& Ballantyne 2015). This hypothesis is also supported by the value of the reflection parameter $R_{\text {pex }} \simeq 0$ obtained in the spectral analysis, describing a torus which covers only a small fraction of the space surrounding the AGN. Obscuring material can represent the main matter reservoir for accreting SMBHs, triggering feedback through nuclear outflows or jet emission, as in the case of RL AGNs. For high Eddington parameters, $\log \lambda>-1.5$ (as in $3 C 215$ ), the covering fraction of the torus is small ( 40\%) because a large fraction of that material was and maybe it is still being accreted by the central SMBH, bringing it to high luminosity values (Ricci et al. 2017). Seyfert 1 galaxies show a similar obscuration but absence of jet emission, possibly substituted by nuclear outflows, producing Fe $\mathrm{K} \alpha \mathrm{EW}$ of the order of $\sim 53 \mathrm{eV}$ (Shu et al. 2010).

The characteristics described in this scenario may appear to be in disagreement with what was observed in the IR band of 3C 215 from Runnoe et al. (2013). These authors found a covering fraction related to the MIR radiation $c=64 \%$, which is very large considering that the torus does not show significant presence of dust, which would permit the interaction between the torus and the IR flux. An interpretation of this discrepancy could be the presence of a second obscuring component, such as a polar dusty outflow that is luminous in the IR band (Hönig et al. 2012; Ramos Almeida \& Ricci 2017). Such a wind could account for the large covering factor estimated by previous studies. Nonetheless, it does not contradict our results regarding the obscuring torus obtained with our X-ray analysis.

\subsection{The possibility of a slim disk}

As discussed in Sect. 5.2, the lack of reflection features in the internal region of this radio-loud quasar may possibly stand in agreement with a double-state of the accretion disk, where the outer portion shows a standard Shakura-Sunyaev geometry (Shakura \& Sunyaev 1973), while the internal one $\left(R_{\text {in }} \lesssim 38 R_{\mathrm{g}}\right)$ is radiatively inefficient and geometrically thick, favoring the production of a jet. Nonetheless, ADAF states are present only in the disk of low accreting SMBHs. If the Eddington parameter is high $(\lambda \gtrsim 0.3)$ the disk becomes thick enough to make advection an efficient cooling mechanism, hence leading to a drop of the disk emissivity. This disk state, related to highly accreting SMBHs, is called a slim disk (Abramowicz et al. 1988; Abramowicz \& Fragile 2013; Yuan \& Narayan 2014); within the uncertainties, it is in agreement with the Eddington parameter estimated here: $\lambda=0.13_{-0.09}^{+0.28}$.

According to this scenario, the presence of a composite accretion disk leads to an anisotropic radiation field that interacts differently with the various components. Consequently, a selfshadowing phenomenon can produce two dynamically distinct 
zones of the BLR, with one of them possibly composed by $\mathrm{H} \beta$ outflowing clouds (Wang et al. 2014), which is in agreement with the previous BLR discussion of 3C 215. The higher the accretion rate, the greater is the self-shadowing effect, hence, we do not expect it to be very intense in this source since it is usually related to super-Eddington SMBHs.

In relation to this local disk emissivity drop, we could expect also a drop of the interaction between the UV incident flux and the torus, leading to a decrease of the reprocessed IR flux and to a torus characterization bias, since it would appear as an inefficient obscurer. Nonetheless, these features are also observed in the X-ray band of $3 \mathrm{C} 215$, hence, they are not due to only the self-shadowing bias. In addition, to account for the intense IR emission of this source we proposed the presence of a second dusty component. In this scenario, this reprocessing component could be constituted by an outflowing BLR that is possibly dustrich (Czerny \& Hryniewicz 2011; Baskin \& Laor 2018).

Although this scenario is able to describe several observational features of $3 \mathrm{C} 215$, it does not provide an explanation of the particular geometry of the jet emission observed in this source, noting the necessity of a possible new scenario, discussed in the next section.

\subsection{The possibility of a SMBH binary system}

A third speculative scenario that sets out to explain our X-ray and previous multi-wavelength observations of 3C 215 involves the presence of a second SMBH that forms a SMBH binary system (SMBHB; Begelman et al. 1980; De Rosa et al. 2019). Various motivations led us to consider the possibility of a SMBHB in describing this source, such as hints of a previous major merger event involving the 3C 215 host galaxy (as we discussed in Sect. 2), the twisted and knotted radio jet emission in this AGN that is possibly due to the formation of warped accretion disks (Bardeen \& Petterson 1975; Papaloizou \& Pringle 1983; Nayakshin 2005; Ulubay-Siddiki et al. 2009), or a direct interaction of the binary with the jet-producing region (Lawrence \& Elvis 2010; Tremaine \& Davis 2014) that leads to the perturbed shape of the obscuring torus and the relatively high accretion rate of this source. All these features can be explained by the presence of a putative secondary SMBH that perturbs the space surrounding its orbit. If the distance between the two SMBHs was at sub-pc scales, this can possibly also explain the lack of material, hence, the reflection features in the internal portion of the accretion disk at distances of $\lesssim 38 R_{\mathrm{g}}$, as observed in the X-ray analysis.

Nonetheless, SMBHBs are very elusive and hard to recognize since they can not be spatially resolved, such that a large fraction of binary system detection has been serendipitous. The state-of-the-art methods to select these systems are multiepoch spectroscopic observations, multi-band follow-ups, and cross-correlation analyses, which have been able to show some signatures of the periodic motion of the binary. Therefore, the current data available on 3C 215 are not enough to firmly select it as a strong SMBHB candidate. Nonetheless, there are two main SMBHBs selection methods that rely only on single-epoch spectroscopic observations of broad emission lines (BELs) in the optical/UV band.

The first method consists of detecting periodical and intense Doppler shift $\Delta v$ of BEL peaks produced in the BLR, linked to the internal orbital motion in the same way spectroscopic binary stars are observed (Bogdanović et al. 2008; Eracleous et al. 2012; Decarli et al. 2013). Here, $\Delta v$ can be computed by the difference between $\mathrm{Mg}_{\mathrm{II}}$ and [OIII] peak velocities, the latter having a good approximation of the same velocity of the host galaxy (Ju et al. 2013; Wang et al. 2017). For 3C 215, the estimate of $\Delta v \simeq 915 \mathrm{~km} \mathrm{~s}^{-1}$ (Tang et al. 2012) is very high compared to the usual values (e.g. Wang et al. 2017) and close to the selection threshold $|\Delta v| \gtrsim 1000 \mathrm{~km} \mathrm{~s}^{-1}$.

The second method is based on the fact that during binary orbital decay, the BLR starts to emerge from the Roche lobes of the binary, producing outflows of material and consequently the BLR erosion. Since this process affects the external region of the BLR more significantly, we could expect for low-ionization emission lines to suffer a significant flux decrease with respect to the high-ionization ones, which are produced more internally. Interestingly, the BLR erosion is consistent with the hypothesis of an outflowing external portion of the BLR proposed in Sect. 5.2. Montuori et al. $(2011,2012)$ found that the ratio between the fluxes of the $\mathrm{C}_{\mathrm{IV}}$ line $\left(F_{\mathrm{CIV}}\right)$ and of the $\mathrm{Mg}_{\mathrm{II}}$ line $\left(F_{\mathrm{MgII}}\right)$ increases with an increasing orbital period, $P$, hence, this ratio constitutes a good binary diagnostic.

The selection of SMBH binary candidates sources is based on their satisfying the relation $F_{\mathrm{MgII}} / F_{\mathrm{CIV}} \lesssim 0.1$, with a threshold that is smaller with respect to the common value, which is $F_{\mathrm{MgII}} / F_{\mathrm{CIV}} \sim 0.3-0.4$. 3C 215 shows a flux ratio of $F_{\mathrm{MgII}} / F_{\mathrm{CIV}}=0.14 \pm 0.04$, which is consistent with the selection requirement. However, these two analyses can only select $3 \mathrm{C} 215$ as a potential weak SMBHB candidate, given that they are not able to provide clear and unequivocal evidence of the presence of the binary through the detection of orbital modulations.

\section{Conclusions}

We performed the most accurate X-ray spectral analysis of the broad line radio galaxy $3 \mathrm{C} 215$ using high quality data from the XMM-Newton satellite. Thanks to a comparison of the results we obtained with multi-wavelength observations from the literature, we were able to characterize the innermost components of this AGN, modeling their geometry and mutual interactions.

This source shows a jet contribution in the X-ray band that can be described as a flat power law, very similarly to FSRQs. The primary X-ray continuum from the corona interacts with a reflector placed at relatively large distances $\left(R \gtrsim 38 R_{\mathrm{g}}\right)$ from the $\mathrm{SMBH}$. Evidence suggests that the reflection component is the accretion disk, which is ionized $\left(\log \xi \simeq 2.31 \mathrm{erg} \mathrm{s}^{-1} \mathrm{~cm}\right)$, and also part of the BLR, which shows a stratified structure, with the $\mathrm{C}_{\mathrm{IV}}$ emission line emitter exhibiting a disk-like geometry over the accretion flow and a putative outflowing $\mathrm{H} \beta$ source region. The ionized disk gas falls in towards the SMBH, which accretes its mass with an high sub-Eddington rate $\left(\lambda=0.13_{-0.09}^{+0.28}\right)$.

Spectral features expected from the putative obscuring torus are very weak, if not altogether absent, and this suggests a patchy geometry with a low covering fraction $(\sim 40 \%)$ and reflection parameter $\left(R_{\text {pex }} \simeq 0\right)$. The low Fe $\mathrm{K} \alpha$ equivalent width $(\mathrm{EW}<41 \mathrm{eV})$ suggests a minimal fraction of dust in the X-ray torus structure, in contrast with previous observations in the literature that reported a covering fraction $c=64 \%$ in the MIR band directly linked with a dusty obscurer. This can be explained by the presence of an internal polar dusty wind that contributes, together with the torus, in the nuclear obscuration process.

We compared the results of multiple spectral analyses carried out in different years and, thus, observed an intense variability on the part of the X-ray spectrum of $3 \mathrm{C} 215$, which is becoming fainter (by almost a factor of 4) with an overall time step of $\sim 17$ years. This variability can be associated with a drop in the 
Eddington parameter that leads to the evaporation of the inner edge of the accretion disk in a radiatively inefficient geometrically thick accretion flow.

We propose three scenarios that could explain these observed properties:

1. An ADAF state in the internal region, which is in accordance with the reflector distance, but not with the accretion rate, as it would be too high for this type of structure.

2. A slim inner disk, which has a similar geometrical structure to that of the ADAF and is consistent with the 3C 215 accretion regime. It accounts for almost all the observed features, except for the unusual jet emission shape.

3. A SMBH binary system, which may describe the overall structure of $3 \mathrm{C} 215$ in a good way, but is still lacking in terms of a firm SMBHB detection, making this scenario particularly speculative.

Some observational features manifested by 3C 215 are common in RL sources, such as weak reflection features (e.g. Bostrom et al. 2014; Ursini et al. 2018b; Ronchini et al. 2019; Chalise et al. 2020). In contrast, RQ AGNs show an opposite behavior, with a standard accretion disk that extends down to the ISCO, and which is able to produce strong reflection features such as the $\mathrm{Fe} \mathrm{K} \alpha$ line and provide good spin parameter estimates (Fabian et al. 2014; Reynolds 2020).

Nonetheless, 3C 215 shows an obscuring torus that is not very different from those in RQ AGNs (Shu et al. 2010; Tazaki et al. 2013; Panessa et al. 2016) and its accretion rate $\lambda$ is high compared to other RL AGNs, as well as being more similar to the one expected in RQ sources. In fact, as we discussed in Sect. 5.4, these two characteristics are strongly linked. However, the accretion disk geometry, which depends on the accretion rate, must be thick enough to allow matter collimation and jet production (Ballantyne 2007). As we discussed, this peculiar geometry can be obtained both with high and low accretion rates, thus suggesting that the most significant phenomenon helping jet production could possibly be the disk height (Abramowicz \& Fragile 2013) or the mutual rotation direction between the SMBH and the accreting material (Garofalo 2019), rather than the accretion rate itself.

This observation allows us to add this contribution to the BLRG catalog and, via the hints of jet emission in the X-ray band, allows us to better understand how heterogeneous these sources are, at least in the X-ray band. Discovering how and why BLRGs show different observational features can be crucial in solving the RL-RQ dichotomy. Unfortunately, with the data obtained through our observations and the previous ones from the literature, we can achieve only a weak SMBHB candidate selection, although the selection tests we made for 3C 215 are promising. New multi-epoch spectroscopic observations, multiband follow-ups, and cross-correlation studies are required in order to firmly determine $3 \mathrm{C} 215$ as a strong SMBH candidate and to possibly add this source in the new developing SMBHB catalogs, which could then be compared with those related to classical single AGNs. In particular, with new observations in the radio band, for example, with the SKA facility, it will be possible to resolve the jet source of 3C 215 with high resolution, thereby acquiring new information about the process that bends its jet. Also, subsequent time monitoring in the opti$\mathrm{cal} / \mathrm{UV}$ band with the HST could provide hints of some kind of time variability in the emission lines, possibly associated with orbital periods of a putative binary system. Finally, an X-ray follow-up can further monitor the X-ray variability observed in this source, providing additional validation for the SMBHB scenario.
Acknowledgements. AM and FT thank the anonymous referee for the constructive comments. This work was performed by AM for his Master Thesis in Physics - Curriculum Astrophysics at the Tor Vergata University of Rome based on observations obtained with XMM-Newton, an ESA science mission with instruments and contributions directly funded by ESA Member States and NASA. AM and FT thank F. Vagnetti, F. Panessa and G. Bruni for the useful discussions.

\section{References}

Abramowicz, M. A., Czerny, B., Lasota, J. P., \& Szuszkiewicz, E. 1988, ApJ, 332,646

Abramowicz, M. A., \& Fragile, P. C. 2013, Liv. Rev. Rel., 16, 1

Ballantyne, D. R. 2007, Mod. Phys. Lett. A, 22, 2397

Ballantyne, D. R., Ross, R. R., \& Fabian, A. C. 2002, MNRAS, 332, L45

Ballantyne, D. R., Bollenbacher, J. M., Brenneman, L. W., et al. 2014, ApJ, 794, 62

Bardeen, J. M., \& Petterson, J. A. 1975, ApJ, 195, L65

Baskin, A., \& Laor, A. 2018, MNRAS, 474, 1970

Begelman, M. C., Blandford, R. D., \& Rees, M. J. 1980, Nature, 287, 307

Bettoni, D., Falomo, R., Fasano, G., \& Govoni, F. 2003, A\&A, 399, 869

Blandford, R. D., \& Znajek, R. L. 1977, MNRAS, 179, 433

Bogdanović, T., Smith, B. D., Sigurdsson, S., \& Eracleous, M. 2008, ApJS, 174, 455

Bostrom, A., Reynolds, C. S., \& Tombesi, F. 2014, ApJ, 791, 119

Bridle, A. H., Hough, D. H., Lonsdale, C. J., Burns, J. O., \& Laing, R. A. 1994, AJ, 108, 766

Chalise, S., Lohfink, A. M., Kara, E., \& Fabian, A. C. 2020, ApJ, 897, 47

Chiaberge, M., \& Marconi, A. 2011, MNRAS, 416, 917

Chiaberge, M., Gilli, R., Lotz, J. M., \& Norman, C. 2015, ApJ, 806, 147

Comastri, A., Fossati, G., Ghisellini, G., \& Molendi, S. 1997, ApJ, 480, 534

Czerny, B., \& Hryniewicz, K. 2011, A\&A, 525, L8

Dauser, T., Garcia, J., Parker, M. L., Fabian, A. C., \& Wilms, J. 2014, MNRAS, 444, L100

Dauser, T., García, J., Walton, D. J., et al. 2016, A\&A, 590, A76

De Rosa, A., Vignali, C., Bogdanović, T., et al. 2019, New Astron. Rev., 86, 101525

Decarli, R., Labita, M., Treves, A., \& Falomo, R. 2008, MNRAS, 387, 1237

Decarli, R., Falomo, R., Treves, A., et al. 2010, MNRAS, 402, 2441

Decarli, R., Dotti, M., Fumagalli, M., et al. 2013, MNRAS, 433, 1492

Duras, F., Bongiorno, A., Ricci, F., et al. 2020, A\&A, 636, A73

Eracleous, M., Sambruna, R., \& Mushotzky, R. F. 2000, ApJ, 537, 654

Eracleous, M., Boroson, T. A., Halpern, J. P., \& Liu, J. 2012, ApJS, 201, 23

Fabian, A. C., Parker, M. L., Wilkins, D. R., et al. 2014, MNRAS, 439, 2307

Fan, J. H., Yang, J. H., Yuan, Y. H., Wang, J., \& Gao, Y. 2012, ApJ, 761, 125

Fernini, I. 2007, AJ, 134, 158

Floyd, D. J. E., Kukula, M. J., Dunlop, J. S., et al. 2004, MNRAS, 355, 196

García, J., Dauser, T., Reynolds, C. S., et al. 2013, ApJ, 768, 146

García, J., Dauser, T., Lohfink, A., et al. 2014, ApJ, 782, 76

Garofalo, D. 2019, ApJ, 876, L20

Garofalo, D., Evans, D. A., \& Sambruna, R. M. 2010, MNRAS, 406, 975

Garofalo, D., Kim, M. I., \& Christian, D. J. 2014, MNRAS, 442, 3097

Gilbert, G. M., Riley, J. M., Hardcastle, M. J., et al. 2004, MNRAS, 351, 845

Gohil, R., \& Ballantyne, D. R. 2015, MNRAS, 449, 1449

Grandi, P., \& Palumbo, G. G. C. 2007, ApJ, 659, 235

Grandi, P., Malaguti, G., \& Fiocchi, M. 2006, ApJ, 642, 113

Hardcastle, M. J., Evans, D. A., \& Croston, J. H. 2006, MNRAS, 370, 1893

Hönig, S. F., Kishimoto, M., Antonucci, R., et al. 2012, ApJ, 755, 149

Ikeda, S., Awaki, H., \& Terashima, Y. 2009, ApJ, 692, 608

Ju, W., Greene, J. E., Rafikov, R. R., Bickerton, S. J., \& Badenes, C. 2013, ApJ, 777,44

Kataoka, J., Stawarz, Ł., Takahashi, Y., et al. 2011, ApJ, 740, 29

Labita, M., Treves, A., Falomo, R., \& Uslenghi, M. 2006, MNRAS, 373, 551

Laor, A. 2000, ApJ, 543, L111

Lawrence, A., \& Elvis, M. 2010, ApJ, 714, 561

Lehnert, M. D., Miley, G. K., Sparks, W. B., et al. 1999, ApJS, 123, 351

Lohfink, A. M., Reynolds, C. S., Mushotzky, R., \& Nowak, M. 2013, AAS/High

Energy Astrophysics Division, 13, 108.11

Márquez, I., Durret, F., \& Petitjean, P. 1999, A\&AS, 135, 83

Moderski, R., Sikora, M., \& Lasota, J. P. 1998, MNRAS, 301, 142

Montuori, C., Dotti, M., Colpi, M., Decarli, R., \& Haardt, F. 2011, MNRAS, 412, 26

Montuori, C., Dotti, M., Haardt, F., Colpi, M., \& Decarli, R. 2012, MNRAS, 425, 1633

Murphy, K. D., \& Yaqoob, T. 2009, MNRAS, 397, 1549

Nandra, K., O’Neill, P. M., George, I. M., \& Reeves, J. N. 2007, MNRAS, 382, 194

Narayan, R., \& Yi, I. 1994, ApJ, 428, L13 
Narayan, R., \& Yi, I. 1995, ApJ, 444, 231

Narayan, R., \& McClintock, J. E. 2008, New Astron. Rev., 51, 733

Nayakshin, S. 2005, MNRAS, 359, 545

Noda, H., \& Done, C. 2018, MNRAS, 480, 3898

Noda, H., Makishima, K., Yamada, S., et al. 2014, ApJ, 794, 2

Noda, H., Minezaki, T., Watanabe, M., et al. 2016, ApJ, 828, 78

Padovani, P. 2017, Nat. Astron., 1, 0194

Padovani, P., Alexander, D. M., Assef, R. J., et al. 2017, A\&ARv, 25, 2

Panessa, F., Barcons, X., Bassani, L., et al. 2007, A\&A, 467, 519

Panessa, F., Bassani, L., Landi, R., et al. 2016, MNRAS, 461, 3153

Papaloizou, J. C. B., \& Pringle, J. E. 1983, MNRAS, 202, 1181

Ramos Almeida, C., \& Ricci, C. 2017, Nat. Astron., 1, 679

Ravindranath, S., Ho, L. C., \& Filippenko, A. V. 2002, ApJ, 566, 801

Reeves, J. N., \& Turner, M. J. L. 2000, MNRAS, 316, 234

Reynolds, C. S. 2020, ARA\&A, submitted [arXiv:2011.08948]

Ricci, C., Trakhtenbrot, B., Koss, M. J., et al. 2017, Nature, 549, 488

Ronchini, S., Tombesi, F., Vagnetti, F., Panessa, F., \& Bruni, G. 2019, A\&A, 625, A26

Runnoe, J. C., Shang, Z., \& Brotherton, M. S. 2013, MNRAS, 435, 3251

Sambruna, R. M. 1997, ApJ, 487, 536
Sambruna, R. M., Barr, P., Giommi, P., et al. 1994, ApJ, 434, 468

Sambruna, R. M., Eracleous, M., \& Mushotzky, R. F. 1999, ApJ, 526, 60

Shakura, N. I., \& Sunyaev, R. A. 1973, A\&A, 24, 337

Shu, X. W., Yaqoob, T., \& Wang, J. X. 2010, ApJS, 187, 581

Tang, B., Shang, Z., Gu, Q., Brotherton, M. S., \& Runnoe, J. C. 2012, ApJS, 201, 38

Tazaki, F., Ueda, Y., Terashima, Y., Mushotzky, R. F., \& Tombesi, F. 2013, ApJ, 772,38

Tremaine, S., \& Davis, S. W. 2014, MNRAS, 441, 1408

Ulubay-Siddiki, A., Gerhard, O., \& Arnaboldi, M. 2009, MNRAS, 398, 535

Urry, C. M., \& Padovani, P. 1995, PASP, 107, 803

Ursini, F., Bassani, L., Panessa, F., et al. 2018a, MNRAS, 481, 4250

Ursini, F., Bassani, L., Malizia, A., et al. 2018b, https://doi .org/10.5281/ zenodo. 1472958

Vestergaard, M., \& Osmer, P. S. 2009, ApJ, 699, 800

Vestergaard, M., \& Peterson, B. M. 2006, ApJ, 641, 689

Wang, J.-M., Qiu, J., Du, P., \& Ho, L. C. 2014, ApJ, 797, 65

Wang, L., Greene, J. E., Ju, W., et al. 2017, ApJ, 834, 129

Wilms, J., Allen, A., \& McCray, R. 2000, ApJ, 542, 914

Yuan, F., \& Narayan, R. 2014, ARA\&A, 52, 529 